\title{
A Study of Operating Reserve Procurance in Power Markets with Application of Insurance Theory: Contract-based v.s. Pool-based Approaches
}

\author{
C. Y. Chan, Student Member, IEEE, Y. X. Ni, S. M., IEEE and Felix F. Wu, Fellow, IEEE
}

\begin{abstract}
In the power markets, the ancillary services are treated as special commodities. Operating reserve is an important commodity in the category of ancillary services, which supports the system reliability against unexpected generator outages. In this paper, we study the procurance of operating reserve via two approaches, i.e. contract-based model and pool-based model. In order to guarantee the availability of reserved capacity and reduce the loss of consumer at generator forced outages, insurance policy is applied. Decentralized optimal decision is adopted. After presenting the math models of the two approaches, it is proven that pool-based approach is more cost-effective and should be adopted in the deregulated power markets.
\end{abstract}

Index Terms-- Ancillary Service, Decentralized Optimization, Deregulated Power Markets, Insurance Theory

\section{INTRODUCTION}

$\mathrm{E}_{\mathrm{s}}^{\mathrm{c}}$ ectricity is a very special product. It is not storable and the demands are fluctuating and lack of elasticity, therefore it is difficult to realize real-time power balance at lower cost. In the power market, not only electricity, but also transmission services and ancillary services are important commodities to be traded. Operating reserve is an important commodity in the category of ancillary services, which supports the system reliability against unexpected generation outages. In traditional power systems, the ancillary services are usually centrally handled. The system operator will determine the reserves requirement and procure it from the reserve suppliers. And the corresponding cost is then allocated to the consumer based on electricity consumption of individual end users.

It is well known that, in conventional power systems, the reserve requirement is determined more on reliability

The research is sponsored by RGC of the H.K. Government, to whom the authors would like to express their heartfelt acknowledgments.

C. Y. Chan is with Dept. of Electrical and Electronic Engineering, The University of Hong Kong, Pokfulam Road, Hong Kong (e-mail: chiychan@eee.hku.hk).

Y. X. Ni is with Dept. of Electrical and Electronic Engineering, The University of Hong Kong, Pokfulam Road, Hong Kong (e-mail: yxni@eee.hku.hk).

Felix F. Wu is with Dept. of Electrical and Electronic Engineering, The University of Hong Kong, Pokfulam Road, Hong Kong (e-mail: ffwu@eee.hku.hk). viewpoint than on cost-effective viewpoint. More often, the N1 security criterion is used, i.e. the reserved capacity should at least be greater than or equal to the largest generation unit, and in the meantime based on experiences the reserved capacity should be no less than a certain percentage of total operation capacity. In the power industry deregulation, the responsibility for and procurance of reserve are studied in quite a few papers [1-5]. However, most of the researches use centralized approaches.

A centralized handling and decision on ancillary service and a uniform pricing scheme definitely cannot reflect the need of the consumers. i.e. consumers have no choices on preferred reliability level and cost-effective operating reserve. Besides there are no incentives for the system operator to make efficient decision on reserved capacity. There are also no incentives for the reserve capacity providers to commit the requested reserve capacity. Therefore, the flexibility, efficiency and quality of the current ancillary service markets are in question. Besides in case of electricity supply interruption, the consumers will suffer great economic risk.

According to the market economy principles, the best way for efficient commodity transaction is to allow decentralized optimal decisions and it makes use of the 'invisible hand' of the market to induce optimal social welfare and market efficiency. In [6], a conceptual study on reserve capacity procurance is conducted with insurance theory applications $[7,8]$. A power insurer is introduced. It represents the consumer (also the insured) to purchase reserve capacity from gencos and sells insurance policy to the consumers.

The results of [6] are very challenging:

- The operating reserve market allows the power consumers to determine their own reserve requirements.

- The decentralized optimal decision becomes possible, and yields the same optimal results as the centralized optimal decision.

- With insurance policy, the consumers can shift the outage risk to the power insurer.

- The insurance policy induces an incentive and enforces the liability for the insurer to manage the reserve capacity.

However, the model used in [6] is very simple and limited 
to one generation, one consumer and one insurer only. In this paper, we will extend the mechanism in [6] to multi-player cases. In order to simplify our analytical study, we assume the studied system has $\mathrm{N}$ identical gencos and $\mathrm{M}$ identical consumers. Two approaches are investigated. One is contractbased approach, and the other pool-based approach. In both cases, N-1 security assumption will hold; i.e. only one generator will outage at a time. The mathematic models and optimal decision procedures are presented. It is proven that pool-based approach is more cost-effective and should be adopted in the deregulated power markets.

This paper is organized as follows. The details of the reserve capacity market mechanism with the contract-based approach are introduced in section II. The market with poolbased approach is studied in section III. In section IV, the comparison is made between the two approaches and it is proven pool-based approach is more cost-effective. Conclusions are drawn in section V.

\section{CONTRACT-BASED APPROACH}

We assume that there are three types of players in the market: $\mathrm{N}$ gencos $\left(G_{i}, i=1 \cdots N\right), \mathrm{M}$ consumers $\left(C_{i, j}, i=1 \cdots N, j=1 \cdots H, M=N \cdot H\right)$ and a power insurer (I). We assume all the $\mathrm{N}$ gencos are identical and the $\mathrm{M}$ consumers are identical as well. The gencos provide both electric energy and reserve capacity on requests. The gencos want to maximize their own profits, so do the consumers. In the electricity market, gencos sell power to consumers at the Market Clearing Price $(M C P)$. The total power supply $S_{\Sigma}$ is assumed to be equal to the total demand $Q_{\Sigma}$ with transmission loss neglected. It is reasonable to assume that genco $i$ maintains an acceptable outage probability $O P_{i}\left(0<O P_{i}<1\right)$ declared in the contract. We can use penalty system to enforce the liability for the genco to maintain $O P_{i}$. In this paper, we assume the penalty system is in well operation, therefore $O P_{i}$ is fixed in our study.

In this section, a contract-based model for reserve procurance will be derived. Assume each genco ( $G_{i}$ ) has bilateral contracts to sell real power to $\mathrm{H}$ consumers denoted as group $i$ (see Fig. 1.). $C_{i, j}$ denotes consumer $\mathrm{j}$ of group $i$ with bilateral contract with genco $i$.

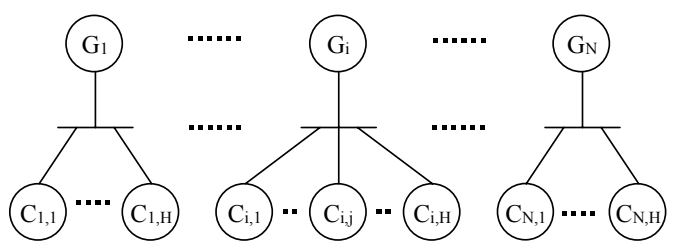
$M=N \cdot H$

Fig. 1. Contract-based model for operating reserves

The consumers can obtain benefits via purchase real power, but they will suffer loss (outage cost $O C_{i, j}$ ) when the outage occurs. Assuming that the consumer's benefit via utilizing power $Q_{i, j}$, is $B_{i, j}\left(Q_{i, j}\right)$. The function $B_{i, j}\left(Q_{i, j}\right)$ usually has the following characteristics: $\left((.)^{\prime}=\frac{d(.)}{d Q_{i, j}}\right)$

$$
\begin{array}{ll}
B_{i, j}(0)=0 & \\
B_{i, j}{ }^{\prime}\left(Q_{i, j}\right) \geq 0 & \text { for } Q_{i, j} \geq 0 \\
B_{i, j}{ }^{\prime \prime}\left(Q_{i, j}\right) \leq 0 & \text { for } Q_{i, j} \geq 0
\end{array}
$$

In the contract-based approach, we assume if genco $G_{i}$ outages, the supply to consumers $C_{i, j}, j=1 \cdots H$ who have contracts with $G_{i}$ will be interrupted; while the other consumers should not be affected (although they are all connected to the same network). Therefore, when genco $G_{i}$ outages, the consumer $C_{i, j}$ will lose all fed power $Q_{i, j}$. The outage cost for consumer $C_{i, j}$ with reserve capacity $T_{i, j}$ and the corresponding energy cost in fuel cost nature $\frac{1}{H} C_{R E}\left(T_{i, j} \cdot H\right)$ can be express as

$$
O C_{i, j}\left(T_{i, j}\right)=\left\{\begin{array}{l}
{\left[B_{i, j}\left(Q_{i, j}\right)-M C P \cdot Q_{i, j}\right]} \\
-\left[B_{i, j}\left(T_{i, j}\right)-\frac{1}{H} C_{R E}\left(T_{i, j} \cdot H\right)\right]
\end{array}\right\} \cdot \Delta t
$$

The first term within the bracket is the expected net profit without any outage at known electricity market clearing price (MCP) and the second term within the bracket is the expected net profit when an outage occurs. Because of N-1 security and identical consumer assumptions, the total reserve capacity will be $\left(T_{i, j} \cdot H\right)$, and the corresponding energy $\operatorname{cost}\left(C_{R E}(\cdot)\right)$ will be shared by $\mathrm{H}$ consumers in load group $i$. The capacity cost in (opportunity cost nature), which is constantly paid no matter you use the reserve energy or not, will be considered separatley. The outage cost of consumer $C_{i, j}$ should be zero when genco $G_{k}(k \neq i)$ outages. Therefore, the expected outage cost $E O C_{i, j}\left(T_{i, j}\right)$ for consumer $C_{i, j}$ can be defined as the product of the outage probability and the outage cost cause by the genco $G_{i}$ outage only, i.e.

$$
\operatorname{EOC}_{i, j}\left(T_{i, j}\right)=O P_{i} \cdot O C_{i, j}\left(T_{i, j}\right)
$$

There is an incentive for the consumers to spend certain amount of money to reduce the loss, e.g. to purchase insurance policy. The consumers will also optimize their decisions on $T_{i, j}$ according to the reserve capacity price of reserves and their own outage cost functions.

Assume the power insurer acts as both an insurer and a reserve schedule coordinator concurrently, and he is a nonprofit operator. (There is no difficulty to include the profit.) Without considering the administration cost, his expected profit is assumed to be zero. It is also assumed that the reserve capacity cost curve $C_{R}\left(T_{\Sigma}\right)$ is known, where $T_{\Sigma}$ is systemwide total reserve. The corresponding cost of the reserve capacity is allocated fairly to the consumers based on reserve capacity requested by individual consumers.

The insurance policy for the power consumers is designed 
as follow: once the power consumer $C_{i, j}$ has purchased the insurance policy with reserve capacity of $T_{i, j}$. The power insurer will procure the amount of reserved capacity $R_{i, j}$ from the supplies, and the reserve capacity will be supplied to the consumer $C_{i, j}$ in the event of genco $i$ outage. Consumer $C_{i, j}$ should pay corresponding cost $C_{R}\left(T_{\Sigma}\right) / M$ for the reserved capacity. In the meantime, the consumer can claim a percentage of his outage loss (say $K_{i, j}, 0 \leq K_{i, j} \leq 1$ ) to be compensated by the insurer in case outage happens, however, he must pay a certain premium $p_{i, j}\left(T_{i, j}, K_{i, j}\right)$ for it. The total premium for the insurance policy to be paid by $C_{i, j}$ to the insurer should be ( $M=N \cdot H$, see Fig. 1$)$ :

$$
p_{i, j}\left(T_{i, j}, K_{i, j}\right)=\frac{C_{R}\left(T_{\Sigma}\right)}{M}+K_{i, j} \cdot \operatorname{EOC}_{i, j}\left(T_{i, j}\right), 0 \leq K_{i, j} \leq 1
$$

where $T_{i, j}$ : requested reserve capacity by the consumer $C_{i, j}$, in MW; $K_{i, j}$ : percentage of the outage loss of consumer to be insured; $C_{R}\left(T_{\Sigma}\right)$ : the total cost for the power insurer to procure the total reserved capacity $T_{\Sigma} ; \operatorname{EOC}_{i, j}\left(T_{i, j}\right)$ : expected outage loss of the consumer when the reserved capacity is $T_{i, j}$. The second term is indeed the premium corresponding to the insurance policy itself [8]. Since reference [6] has proved that the optimal decision of each consumer is to purchase the full compensated insurance policy, so $K_{i, j}=1$. With the $\mathrm{N}-1$ security assumption, the rational insurer and consumers know the reserves $T_{\Sigma}$ can be repeatedly used by the $\mathrm{N}$ groups. Meanwhile, for a generator outage, only relevant $\mathrm{H}$ consumers will suffer from the outage in contractbased approach. Therefore, the premium in (4) is

$$
\begin{aligned}
& \left(T_{\Sigma}=T_{i, j} \cdot H\right): \\
& p_{i, j}\left(T_{i, j}\right)=\frac{C_{R}\left(T_{i, j} \cdot H\right)}{M}+E O C_{i, j}\left(T_{i, j}\right)
\end{aligned}
$$

The consumer with the insurance policy has the follow expected-cost minimization problem

$$
\begin{aligned}
& \underset{T_{i, j}}{\operatorname{Min}} T C_{i, j}\left(T_{i, j}\right)=E O C_{i, j}\left(R_{i, j}\right)+p_{i, j}\left(T_{i, j}\right)-E O C_{i, j}\left(R_{i, j}\right) \\
& \operatorname{Min}_{T_{i, j}} T C_{i, j}\left(T_{i, j}\right)=\frac{C_{R}\left(T_{i, j} \cdot H\right)}{M}+O P_{i} \cdot O C_{i, j}\left(T_{i, j}\right)
\end{aligned}
$$

The first term of (6.a) is the expected outage cost when real reserved capacity provided to $C_{i, j}$ is $R_{i, j}$, the second term is the premium paid for the insurance policy and the third term is the expected claim received from the insurer to compensate the outage loss. The optimal $T_{i, j}$ for (6) should satisfy

$$
\begin{aligned}
\frac{\partial T C_{i, j}\left(T_{i, j}\right)}{\partial T_{i, j}} & =0 \text {, i.e. } \frac{H}{M} \cdot C_{R}{ }^{\prime}\left(T_{i, j} \cdot H\right)+O P_{i} \cdot O C_{i, j}{ }^{\prime}\left(T_{i, j}\right)=0 \\
& \Rightarrow C_{R}{ }^{\prime}\left(T_{i, j} \cdot H\right)=-N \cdot O P_{i} \cdot O C_{i, j}{ }^{\prime}\left(T_{i, j}\right)
\end{aligned}
$$

The L.H.S of (7) is the marginal cost of reserves and the R.H.S. of (7) is the marginal reducing of the expected outage cost.

The purchase of insurance policies by $\mathrm{M}$ consumers is according to their outage cost function (see (2)). However, the power insurer might not buy total reserve capacity from gencos at requested $T_{\Sigma}$. Indeed the insurer is facing the follow expected-cost minimization problem with the real reserve capacity $R_{\Sigma}\left(R_{\Sigma}=R_{i, j} \cdot H\right)$ to be optimized:

$$
\begin{aligned}
\underset{R_{\Sigma}}{\operatorname{Min}} C_{i n s}\left(R_{\Sigma}\right) & =C_{R}\left(R_{\Sigma}\right)+\sum_{i, j} E O C_{i, j}\left(R_{i, j}\right)-\sum_{i, j} p_{i, j}\left(T_{i, j}\right) \\
& =C_{R}\left(R_{\Sigma}\right)+M \cdot O P_{i} \cdot O C_{i, j}\left(R_{i, j}\right)-M \cdot p_{i, j}\left(T_{i, j}\right)
\end{aligned}
$$

where the first term is the total cost to procure the total reserves, the second term is the total expected claim pay to the consumers for compensating their loss at genco outages and the third term is the total premium received from the consumers. Substituting (5) into (8), the optimal $R_{\Sigma}$ for (8) should satisfy $\left(R_{i, j}=R_{\Sigma} / H\right)$ :

$$
\begin{aligned}
\frac{\partial C_{i n s}\left(R_{\Sigma}\right)}{\partial R_{\Sigma}} & =0 \text {, i.e. } C_{R}{ }^{\prime}\left(R_{i, j} \cdot H\right)+M \cdot O P_{i} \cdot O C_{i, j}{ }^{\prime}\left(R_{i, j}\right) \cdot \frac{1}{H}=0 \\
& \Rightarrow C_{R}{ }^{\prime}\left(R_{i, j} \cdot H\right)=-N \cdot O P_{i} \cdot O C_{i, j}{ }^{\prime}\left(R_{i, j}\right)
\end{aligned}
$$

Comparing (7) and (9) we can see that the optimal reserve $R_{i, j}$ maintained by the insurer is exactly the same as optimal reserve $T_{i, j}$ requested by the consumer $C_{i, j}$, which is very attractive property of the approach. And $C_{\text {ins }}\left(R_{\Sigma}\right)=0$ when $R_{\Sigma}=T_{\Sigma}$, since the power insurer is non-profit operator.

If the centralized decision approach is used, the systemwide expected cost $T C_{C B}\left(R_{\Sigma}\right)$ minimization without insurance policy is $\left(R_{\Sigma}=R_{i, j} \cdot H\right)$ :

$$
\operatorname{Min}_{R_{\Sigma}} T C_{C B}\left(R_{\Sigma}\right)=C_{R}\left(R_{\Sigma}\right)+M \cdot O P_{i} \cdot O C_{i, j}\left(R_{i, j}\right)
$$

the centralized optimal solution $R_{\Sigma}$ can be solved for using the first order condition:

$$
\begin{aligned}
\frac{\partial T C_{C B}\left(R_{\Sigma}\right)}{\partial R_{\Sigma}} & =0 \text {, i.e. } C_{R}{ }^{\prime}\left(R_{\Sigma}\right)+M \cdot O P_{i} \cdot O C_{i, j}{ }^{\prime}\left(R_{i, j}\right) \cdot \frac{1}{H}=0 \\
& \Rightarrow C_{R}{ }^{\prime}\left(R_{\Sigma}\right)=-N \cdot O P_{i} \cdot O C_{i, j}{ }^{\prime}\left(R_{i, j}\right)
\end{aligned}
$$

We can see that the decentralized decision approach has the same optimal solution (see (7) and (9)) as the centralized decision (see (11)).

In this section, the contract-based approach for operating reserve has been introduced. For each genco outage, we assume only a small group of consumers will suffer the loss. With the $\mathrm{N}-1$ assumption, the reserves can be repeatedly used.

\section{POOL-BASED APPROACH}

In this section, a pool-based approach for operating reserve will be considered. Still there are $\mathrm{N}$ identical gencos $\left(G_{i}, i=1 \cdots N\right), \mathrm{M}$ identical consumers $\left(C_{j}, j=1 \cdots M\right)$ and a power insurer $(I)$ in the market (see Fig. 2). The characteristics of those participants are same as described in the previous section. However in the pool-based approach, for each genco outage, all the consumers in the pool will lose a small amount of power supply. 


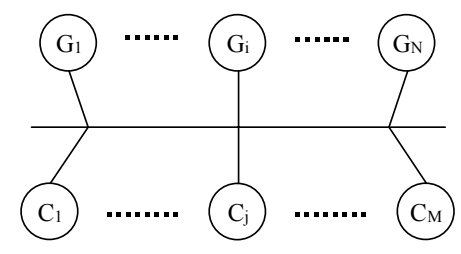

Fig. 2. Pool-based model for operating reserves

Therefore, when genco $G_{i}$ outages, the outage cost for consumer $C_{j}$ can be expressed as

$$
O C_{j}\left(S_{i}, T_{j}\right)=\left\{\begin{array}{l}
{\left[B_{j}\left(Q_{j}\right)-M C P \cdot Q_{j}\right]} \\
{\left[\begin{array}{l}
B_{j}\left(Q_{j}-\frac{Q_{j}}{Q_{\Sigma}} S_{i}+T_{j}\right) \\
-M C P \cdot\left(Q_{j}-\frac{Q_{j}}{Q_{\Sigma}} S_{i}\right)-\frac{1}{M} \cdot C_{R E}\left(T_{j} \cdot M\right)
\end{array}\right]}
\end{array}\right] \cdot \Delta t
$$

where $\quad S_{\Sigma}=\sum_{i} S_{i}=N \cdot S_{i} \quad: \quad$ total supply,

$Q_{\Sigma}=\sum_{j} Q_{j}=M \cdot Q_{j}:$ total demand, $T_{j}:$ reserve capacity of consumer $\mathrm{j}$, and $S_{\Sigma}=Q_{\Sigma} \cdot C_{R E}\left(T_{j} \cdot M\right)$ is the energy cost for total reserve $T_{\Sigma}=T_{j} \cdot M$.

We can simplify the outage cost function as

$$
O C_{j}\left(T_{j}\right)=\left\{\begin{array}{l}
{\left[B_{j}\left(Q_{j}\right)-M C P \cdot Q_{j}\right]} \\
{\left[\begin{array}{l}
B_{j}\left(Q_{j}-\frac{Q_{j}}{N}+T_{j}\right) \\
-M C P \cdot\left(Q_{j}-\frac{Q_{j}}{N}\right)-\frac{1}{M} C_{R E}\left(T_{j} \cdot M\right)
\end{array}\right]}
\end{array}\right\} \cdot \Delta t
$$

The expected outage cost $E O C_{j}\left(T_{j}\right)$ for consumer $C_{j}$ is defined as the summation of the products of outage probability $O P_{i}$ and the outage cost cause by genco $G_{i}$ outage for $i=1, \cdots, N$, i.e..

$$
E O C_{j}\left(T_{j}\right)=\sum_{i} O P_{i} \cdot O C_{j}\left(T_{j}\right)=N \cdot O P_{i} \cdot O C_{j}\left(T_{j}\right)
$$

Base on the assumption of identical gencos and consumers, with insurance policy, the premium function of consumer $\mathrm{j}$ in the pool model can be expressed as $\left(T_{\Sigma}=T_{i, j} \cdot M\right)$

$$
\begin{aligned}
p_{j}\left(T_{j}\right) & =\frac{C_{R}\left(T_{\Sigma}\right)}{M}+E O C_{j}\left(T_{j}\right) \\
& =\frac{C_{R}\left(T_{j} \cdot M\right)}{M}+N \cdot O P_{i} \cdot O C_{j}\left(T_{j}\right)
\end{aligned}
$$

The consumer with the insurance policy has the follow expected-cost minimization problem similar to (6):

$$
\operatorname{Min}_{T_{j}} T C_{j}\left(T_{j}\right)=E O C_{j}\left(R_{j}\right)+p_{j}\left(T_{j}\right)-E O C_{j}\left(R_{j}\right)
$$

Substituting (15) into (16), yield:

$$
\operatorname{Min}_{T_{j}} T C_{j}\left(T_{j}\right)=\frac{C_{R}\left(T_{j} \cdot M\right)}{M}+N \cdot O P_{i} \cdot O C_{j}\left(T_{j}\right)
$$

The optimal $T_{j}$ for (17) should satisfy

$$
\begin{aligned}
& \frac{\partial T C_{j}\left(T_{j}\right)}{\partial T_{j}}=0, \text { i.e. } C_{R}^{\prime}\left(T_{j} \cdot M\right)+N \cdot O P_{i} \cdot O C_{j}^{\prime}\left(T_{j}\right)=0 \\
& \Rightarrow>C_{R}^{\prime}\left(T_{j} \cdot M\right)=-N \cdot O P_{i} \cdot O C_{j}^{\prime}\left(T_{j}\right)
\end{aligned}
$$

$T_{j}$ can be solve for from (18), where the marginal cost of reserve capacity equals to the marginal reducing of the expected outage cost. However, the power insurer might not keep total reserve capacity at requested $T_{\Sigma}=T_{j} \cdot M$. Indeed insurer is facing the follow expected-cost minimization problem similar to (8) with the real reserve capacity $R_{\Sigma}$ to be optimized $\left(R_{\Sigma}=R_{j} \cdot M\right)$

$$
\begin{aligned}
\operatorname{Min}_{R_{\Sigma}} C_{i n s}\left(R_{\Sigma}\right) & =C_{R}\left(R_{j} \cdot M\right)+\sum_{j} E O C_{j}\left(R_{j}\right)-\sum_{j} p\left(T_{j}\right) \\
& =C_{R}\left(R_{j} \cdot M\right)+M \cdot N \cdot O P_{i} \cdot O C_{j}\left(R_{j}\right)-M \cdot p\left(T_{j}\right)
\end{aligned}
$$

The optimal $R_{\Sigma}$ for (19) should satisfy

$$
\begin{aligned}
& \frac{\partial C_{\text {ins }}\left(R_{\Sigma}\right)}{\partial R_{\Sigma}}=0 \text {, i.e. } C_{R}^{\prime}\left(R_{j} \cdot M\right)+M \cdot N \cdot O P_{i} \cdot O C_{j}^{\prime}\left(R_{j}\right) \cdot \frac{1}{M}=0 \\
& \Rightarrow C_{R}^{\prime}\left(R_{j} \cdot M\right)=-N \cdot O P_{i} \cdot O C_{j}^{\prime}\left(R_{j}\right)
\end{aligned}
$$

Comparing (18) and (20) we can see that the optimal reserve $R_{j}$ maintained by the insurer will be the same as the optimal reserve $T_{j}$ requested by the consumer $C_{j}$. And $C_{\text {ins }}\left(R_{\Sigma}\right)=0$ when $R_{\Sigma}=T_{\Sigma}$, since the power insurer is nonprofit operator.

If the centralized decision approach is used, the social expected cost $T C_{P o o l}\left(R_{\Sigma}\right)$ minimization without insurance policy is $\left(R_{\Sigma}=R_{j} \cdot M\right)$

$$
\underset{R_{\Sigma}}{\operatorname{Min}} T C_{\text {Pool }}\left(R_{\Sigma}\right)=C_{R}\left(R_{j} \cdot M\right)+M \cdot N \cdot O P_{i} \cdot O C_{j}\left(R_{j}\right)
$$

The centralized optimal solution $R_{\Sigma}$ can be solved for from (21) :

$$
\begin{aligned}
\frac{\partial T C_{P_{o o l}}\left(R_{\Sigma}\right)}{\partial R_{\Sigma}} & =0 \text {, i.e. } C_{R}{ }^{\prime}\left(R_{j} \cdot M\right)+M \cdot N \cdot O P_{i} \cdot O C_{j}^{\prime}\left(R_{j}\right) \cdot \frac{1}{M}=0 \\
& \Rightarrow C_{R}^{\prime}\left(R_{j} \cdot M\right)=-N \cdot O P_{i} \cdot O C_{j}^{\prime}\left(R_{j}\right)
\end{aligned}
$$

Comparing (18) and (20) with (22), we can conclude that with the insurance policy the decentralized optimal decision yields the same optimal solution as the centralized optimal decision.

\section{COMPARISON OF THE TWO APPROACHES}

In the previous two sections, we have shown that, with a well-defined insurance policy global optimal results can be reached via decentralized decisions, in which each market participant tries to maximize its own benfit.

Because of the difference between the outage cost functions under the contract-based model and the pool-based model, for the same amount of reserve, the two models will have different total costs for reserves, i.e. $O C_{i, j}\left(\frac{R_{\Sigma}}{H}\right)$ of each consumer in contract-based approach is not equal to $O C_{j}\left(\frac{R_{\Sigma}}{M}\right)$ of each consumer in pool-based approach, and

$$
T C_{C B}\left(R_{\Sigma}\right) \neq T C_{\text {Pool }}\left(R_{\Sigma}\right)
$$

Let $R_{\Sigma}{ }^{*}$ be the optimal solution under the contract-based 
model and $R_{\Sigma}{ }^{\Delta}$ be the optimal solution under the pool-based model. The two optimal results will not equal to each other. A significant question is that which approach can induce a cheaper total expected cost. The conclusion is the pool-based approach is more cost-effective, which is proven below.

Let the function $\triangle T C\left(R_{\Sigma}\right)$ be the difference between the total cost function under contract-based approach $T C_{C B}\left(R_{\Sigma}\right)$ and the total cost function under the pool-based approach $T C_{\text {Pool }}\left(R_{\Sigma}\right)$.

$$
\Delta T C\left(R_{\Sigma}\right)=T C_{C B}\left(R_{\Sigma}\right)-T C_{\text {Pool }}\left(R_{\Sigma}\right)
$$

According to the total cost functions in (10) and (21), we have

$$
\begin{aligned}
\Delta T C\left(R_{\Sigma}\right)= & {\left[C_{R}\left(R_{\Sigma}\right)+M \cdot O P_{i} \cdot O C_{i, j}\left(R_{i, j}\right)\right] } \\
& -\left[C_{R}\left(R_{\Sigma}\right)+M \cdot N \cdot O P_{i} \cdot O C_{j}\left(R_{j}\right)\right] \\
& =M \cdot O P_{i} \cdot\left[O C_{i, j}\left(R_{i, j}\right)-N \cdot O C_{j}\left(R_{j}\right)\right]
\end{aligned}
$$

Based on the outage cost functions (2) and (12), (25) will be:

$$
\left.\Delta T C\left(R_{\Sigma}\right)=M \cdot O P_{i} \cdot\left[\begin{array}{l}
\left\{\begin{array}{l}
{\left[B_{i, j}\left(Q_{i, j}\right)-M C P \cdot Q_{i, j}\right]} \\
-\left[B_{i, j}\left(T_{i, j}\right)-\frac{1}{H} C_{R E}\left(T_{i, j} \cdot H\right)\right]
\end{array}\right\} \cdot \Delta t \\
-N \cdot\left\{\begin{array}{l}
{\left[B_{j}\left(Q_{j}\right)-M C P \cdot Q_{j}\right]} \\
{\left[B_{j}\left(Q_{j}-\frac{Q_{j}}{Q_{\Sigma}} S_{i}+R_{j}\right)\right.} \\
-M C P \cdot\left(Q_{j}-\frac{Q_{j}}{Q_{\Sigma}} S_{i}\right)-\frac{1}{M} C_{R E}\left(T_{j} \cdot M\right)
\end{array}\right]
\end{array}\right] \cdot \Delta t\right\}
$$

Under the same electricity market conditions, we know:

$$
\begin{aligned}
& B_{i, j}\left(Q_{i, j}\right)=B_{j}\left(Q_{j}\right), Q_{i, j}=Q_{j}, Q_{\Sigma}=S_{\Sigma} \\
& R_{\Sigma}=R_{i, j} \cdot H, R_{\Sigma}=R_{j} \cdot M, T_{i, j}=R_{i, j}, T_{j}=R_{j}, T_{i, j} \cdot H=T_{j} \cdot M
\end{aligned}
$$

Therefore we can simplify (26) to

$$
\Delta T C\left(R_{\Sigma}\right)=M \cdot O P_{i} \cdot \Delta t \cdot\left[\begin{array}{l}
B_{j}\left(Q_{j}\right)-B_{j}\left(\frac{R_{\Sigma}}{H}\right) \\
-N \cdot B_{j}\left(Q_{j}\right)+N \cdot B_{j}\left(Q_{j}-\frac{Q_{j}}{N}+\frac{R_{\Sigma}}{M}\right)
\end{array}\right]
$$

At $R_{\Sigma}=0$,

$$
\begin{aligned}
\Delta T C(0) & =M \cdot O P_{i} \cdot \Delta t \cdot\left[B_{j}\left(Q_{j}\right)-N \cdot B_{j}\left(Q_{j}\right)+N \cdot B_{j}\left(Q_{j}-\frac{Q_{j}}{N}\right)\right] \\
& =N \cdot M \cdot O P_{i} \cdot \Delta t \cdot\left[-B_{j}\left(Q_{j}\right)\left(1-\frac{1}{N}\right)+B_{j}\left(Q_{j}\left(1-\frac{1}{N}\right)\right)\right]
\end{aligned}
$$

From Lemma 1 (see appendix) equation (A.2) and since $0 \leq a=1-\frac{1}{N} \leq 1 \quad(N \geq 1)$, we have:

$$
\begin{aligned}
& B_{j}\left(Q_{j}\right)\left(1-\frac{1}{N}\right) \leq B_{j}\left(Q_{j}\left(1-\frac{1}{N}\right)\right) \\
& =B_{j}\left(Q_{j}\left(1-\frac{1}{N}\right)\right)-B_{j}\left(Q_{j}\right)\left(1-\frac{1}{N}\right) \geq 0 \\
& =>\Delta T C(0) \geq 0
\end{aligned}
$$

With the N-1 security assumption, the maximum amount of reserve $R_{\Sigma}$ is equal to the power supplied by the largest generation unit, i.e. $R_{\Sigma}=Q_{j}=\frac{Q_{\Sigma}}{N}$, then

$$
\begin{aligned}
& \Delta T C\left(\frac{Q_{\Sigma}}{N}\right)=M \cdot O P_{i} \cdot \Delta t \cdot\left[\begin{array}{l}
B_{j}\left(Q_{j}\right)-B_{j}\left(\frac{Q_{\Sigma}}{N \cdot H}\right)-N \cdot B_{j}\left(Q_{j}\right) \\
+N \cdot B_{j}\left(Q_{j}-\frac{Q_{j}}{N}+\frac{Q_{\Sigma}}{N \cdot M}\right)
\end{array}\right] \\
&=0
\end{aligned}
$$

The first derivative of the $\Delta T C\left(R_{\Sigma}\right)$ is

$$
\begin{aligned}
& \Delta T C^{\prime}\left(R_{\Sigma}\right)=M \cdot O P_{i} \cdot \Delta t \cdot\left[\begin{array}{l}
-B_{j}^{\prime}\left(\frac{R_{\Sigma}}{H}\right) \cdot \frac{1}{H} \\
+N \cdot B_{j}^{\prime}\left(Q_{j}-\frac{Q_{j}}{N}+\frac{R_{\Sigma}}{M}\right) \cdot \frac{1}{M}
\end{array}\right] \\
& =\frac{M}{H} \cdot O P_{i} \cdot \Delta t \cdot\left[B_{j}^{\prime}\left(Q_{j}-\frac{Q_{j}}{N}+\frac{R_{\Sigma}}{M}\right)-B_{j}^{\prime}\left(\frac{R_{\Sigma}}{H}\right)\right]
\end{aligned}
$$

From Lemma 2 (see appendix) equation (A.7), we know:

$$
\begin{array}{ll}
Q_{j}-\frac{Q_{j}}{N}+\frac{R_{\Sigma}}{M} \geq \frac{R_{\Sigma}}{H} & \\
\Rightarrow B_{j}^{\prime}\left(Q_{j}-\frac{Q_{j}}{N}+\frac{R_{\Sigma}}{M}\right) \leq B_{j}^{\prime}\left(\frac{R_{\Sigma}}{H}\right) & \because B_{j}^{\prime \prime}(.) \leq 0 \\
\Rightarrow & \Delta T C^{\prime}\left(R_{\Sigma}\right) \leq 0
\end{array}
$$

Based on equations (30), (31) and (33), we know $\Delta T C(0) \geq 0, \Delta T C\left(\frac{Q_{\Sigma}}{N}\right)=0, \Delta T C^{\prime}\left(R_{\Sigma}\right) \leq 0$ for $0 \leq R_{\Sigma} \leq \frac{Q_{\Sigma}}{N}$

which means $\Delta T C\left(R_{\Sigma}\right) \geq 0$ for all $0 \leq R_{\Sigma} \leq \frac{Q_{\Sigma}}{N}$, or say

$$
T C_{C B}\left(R_{\Sigma}\right) \geq T C_{P o o l}\left(R_{\Sigma}\right) \text { for all } 0 \leq R_{\Sigma} \leq \frac{Q_{\Sigma}}{N}
$$

Denoting $R_{\Sigma}{ }^{*}$ as the optimal value of $R_{\Sigma}$ under the contract-based approach, and $R_{\Sigma}{ }^{\Delta}$ as the optimal value of $R_{\Sigma}$ under the pool-based approach, it is clear:

$$
\begin{array}{cr}
T C_{C B}\left(R_{\Sigma}{ }^{*}\right) \leq T C_{C B}\left(R_{\Sigma}\right) & \text { for all } R_{\Sigma}, 0 \leq R_{\Sigma} \leq \frac{Q_{\Sigma}}{N} \\
T C_{P o o l}\left(R_{\Sigma}{ }^{\Delta}\right) \leq T C_{P o o l}\left(R_{\Sigma}\right) & \text { for all } R_{\Sigma}, 0 \leq R_{\Sigma} \leq \frac{Q_{\Sigma}}{N}
\end{array}
$$

From Eqs. (34) and (35), we can conclude: (set $R_{\Sigma}=R_{\Sigma}{ }^{*}$ in (34))

$$
\begin{aligned}
& T C_{C B}\left(R_{\Sigma}{ }^{*}\right) \geq T C_{\text {Pool }}\left(R_{\Sigma}{ }^{*}\right) \geq T C_{\text {Pool }}\left({R_{\Sigma}}^{{ }^{4}}\right) \\
& \Rightarrow T C_{C B}\left(R_{\Sigma}^{*}\right) \geq T C_{\text {Pool }}\left(R_{\Sigma}{ }^{\Delta}\right)
\end{aligned}
$$

Equation (36) shows the optimal total cost under the contract-based approach will be greater than or equal to the optimal total cost under the pool-based approach, no matter it is centralized optimal decision or decentralized optimal decision (we have proven they yield same value in our designed mechanism). In the other word, the pool-based approach is more cost-effective and should be adopted in the deregulated power markets.

\section{CONCLUSION}

In this paper, conceptual study on decentralized operating reserve plan is conducted. Two possible approaches are suggested. They are contract-based approach and pool-based approach. It is shown that, with the insurance policy, the decentralized optimal decision becomes possible and it yields the same optimal results as the centralized optimal decision in 
both approaches.

It is proven for any amount of reserved capacity, the optimal total cost under the contract-based approach will be greater than or equal to the optimal total cost under the poolbased approach. Therefore, the pool-based approach is more cost-effective and should be adopted in the deregulated power markets.

Further research work is required to consider non-identical multi-players and transmission network impacts. For a large system with a large number of participants, N-1 security assumption may not be adequate. Reserve capacity based on an optimal-percentage of operating capacity should be considered as well.

\section{APPENDIX}

Lemma 1: For a function $f(x)$ with the follow characteristics

$$
\begin{aligned}
& f(0)=0 \\
& f^{\prime}(x) \geq 0, \text { for } x \geq 0 \\
& f^{\prime \prime}(x) \leq 0, \text { for } x \geq 0
\end{aligned}
$$

It can be proven:

$$
\begin{array}{ll}
a \cdot f(x) \geq f(a \cdot x) & \text { for } \forall a \geq 1 \text {; and } \\
a \cdot f(x) \leq f(a \cdot x) & \text { for } \forall 0 \leq a \leq 1
\end{array}
$$

\section{Proof:}

By Taylor series, we have (if $O\left(x^{3}\right) \approx 0$ ):

$$
\begin{aligned}
& a \cdot f(x)=a \cdot f(0)+a \cdot x \cdot f^{\prime}(0)+a \cdot \frac{x^{2}}{2} \cdot f^{\prime \prime}(0) \\
& f(a \cdot x)=f(0)+a \cdot x \cdot f^{\prime}(0)+\frac{a^{2} \cdot x^{2}}{2} \cdot f^{\prime \prime}(0)
\end{aligned}
$$

because $f^{\prime \prime}(x) \leq 0$, we can have

$$
\text { (1) } \begin{aligned}
& a \cdot \frac{x^{2}}{2} \cdot f^{\prime \prime}(0) \geq \frac{a^{2} \cdot x^{2}}{2} \cdot f^{\prime \prime}(0) \text { for } a \geq 1 \\
& =>a \cdot f(x) \geq f(a \cdot x) \text { for } a \geq 1 \\
\text { (2) } & a \cdot \frac{x^{2}}{2} \cdot f^{\prime \prime}(0) \leq \frac{a^{2} \cdot x^{2}}{2} \cdot f^{\prime \prime}(0) \text { for } 0 \leq a \leq 1 \\
\Rightarrow & a \cdot f(x) \leq f(a \cdot x) \text { for } 0 \leq a \leq 1
\end{aligned}
$$

Lemma 2: In the pool approach,

$$
Q_{j}-\frac{Q_{j}}{N}+\frac{R_{\Sigma}}{M} \geq \frac{R_{\Sigma}}{H} \text { if } N, M \geq 1 \text { and } Q_{\Sigma} \geq R_{\Sigma} \cdot N
$$

\section{Proof:}

$$
\begin{aligned}
Q_{j}-\frac{Q_{j}}{N}+\frac{R_{\Sigma}}{M}-\frac{R_{\Sigma}}{H} & =\frac{Q_{\Sigma}}{M}-\frac{Q_{\Sigma}}{M \cdot N}+\frac{R_{\Sigma} \cdot N}{M \cdot N}-\frac{R_{\Sigma} \cdot N}{H \cdot N} \\
& =\frac{1}{M}\left(Q_{\Sigma}-R_{\Sigma} \cdot N\right)-\frac{1}{M \cdot N}\left(Q_{\Sigma}-R_{\Sigma} \cdot N\right) \\
& \geq 0 \quad\left(\because N, M \geq 1, Q_{\Sigma} \geq R_{\Sigma} \cdot N\right) \\
=>Q_{j}-\frac{Q_{j}}{N}+\frac{R_{\Sigma}}{M} \geq \frac{R_{\Sigma}}{H} &
\end{aligned}
$$

\section{REFERENCES}

[1] R. Billinton; N.A. Chowdhury. Operating reserve assessment in interconnected generating systems. IEEE Transactions on Power Systems Vol. 3, No. 4, Nov. 1988, pp. 1479-1487

[2] Terje Gjengedal, Jan Ove Gjerde, Roger Flolo. Ancillary Services in Deregulated Power Systems; What are they; Who Need Them and Who
Pays. Proceedings of International Conference on Power System Technology (POWERCON), 1998. vol. 1. pp. 704-709

[3] Shams N. Siddiqi; Martin L. Baughman. Reliability Differentiated Pricing of Spinning Reserve. IEEE Transactions on Power Systems, Vol. 10, No. 3, Aug. 1995. pp. 1211-1218.

[4] Chung-Li Tseng; Shmuel S. Oren; Alva J. Svoboda; Raymond B. Johnson. Price-based adaptive spinning reserve requirements in power system scheduling. Electrical Power and Energy Systems, Vol. 21, Issue. 2 (1999), pp. 137-145.

[5] E.H. Allen; M.D. llić. Reserve Market for Power Systems Reliability. IEEE Transactions On Power Systems, Volume 15, No. 1, Feb. 2000. pp. 228-233.

[6] C.Y. Chan; F.F. Wu; Y. Ni. Conceptual Study on Decentralized Ancillary Service Plan with Applications of Insurance Theory.

[7] M. Manove. Provider Insurance. Bell Journal of Economics, 1983, pp. 327-330.

[8] Karl H. Borch, The mathematical theory of insurance: an annotated selections of papers on insurance published, 1960-1972 . Lexington, Mass. : Heath, 1974

\section{BIOGRAPHIES}

Chi Yiu Chan (Student M. IEEE) He received his B Sc., M. Eng. degree from The University of Auckland in New Zealand. He is now a Ph.D. candidate of the University of Hong Kong. His research interests are power system reliability, operating reserves requirement and pricing in power systems and power markets.

Felix F. Wu (Fellow, IEEE) He received his Ph. D. degree from University of California at Berkeley (UCB). He is now a chair professor of electrical engineering and vice president of the University of Hong Kong. Prior to that he was a professor and vice-chair of Dept. of EECS, UCB. His research interests are electric energy industry restructuring, power system investment planning, design of modern control centres, distribution automation, distributed processing etc.

Yixin Ni (S. M. IEEE) She received her B. Eng., M. Eng., and Dr. Eng. degrees all in electrical engineering, Tsinghua University, China. She was former professor and director of National Power System Lab, Tsinghua University and now with the University of Hong Kong. Her research interests are power system stability and control, FACTS, AI tech. applications in power systems and power markets. 